\title{
Apply Genetic Algorithm to Minimize the Overkills in Wafer Probe Testing
}

\author{
Shih-Cheng Horng \\ Department of Computer Science \& Information \\ Engineering, Chaoyang University of Technology \\ Taichung, Taiwan, R.O.C. \\ schong@cyut.edu.tw
}

\author{
Han-Tang Tsou \\ Department of Computer Science \& Information \\ Engineering, Chaoyang University of Technology \\ Taichung, Taiwan, R.O.C. \\ s9627618@cyut.edu.tw
}

\begin{abstract}
In this paper, an ordinal optimization (OO) based algorithm is applied to minimize the overkills under a tolerable level of re-probes in a wafer probe testing process, which is formulated as a constrained stochastic simulation optimization problem that consists of a huge input-variable space formed by the vector of threshold values in the testing process. First, we construct a crude but effective model based on a shorter stochastic simulation with a small amount of test wafers. This crude model will then be used as a fitness function evaluation in the genetic algorithm to select $N$ good enough solutions. Then, starting from the selected $N$ good enough solutions we proceed with the goal softening searching procedures to search for a good enough solution. Applying to a real semiconductor product, the vector of good enough threshold values obtained by the proposed algorithm is promising in the aspects of solution quality and computational efficiency. We also demonstrate the computational efficiency of the proposed algorithm by comparing with the genetic algorithm and the evolution strategy.
\end{abstract}

Keywords - wafer probe testing, genetic algorithm, ordinal optimization, overkill, re-probe.

\section{INTRODUCTION}

The wafer fabrication process is a sequence of hundreds of different process steps, which results in an unavoidable variability accumulated from the small variations of each process step. Chips are tested multiple times throughout the design and manufacturing process to ensure the integrity of the chip design and the quality of the manufacturing process. Thus, to avoid incurring the significant expense of assembling and packaging chips that do not meet specifications, a wafer probe testing in the manufacturing process becomes an essential step to identify flaws early [1]. The major components of a wafer probe testing system include probes, probe card, probe station, and test equipment. Wafer probe testing establishes a temporary electrical contact between test equipment and each individual die on a wafer to determine the goodness of a die. In general, an 8-inches wafer may consist of 500 to 15000 dies and each die is a chip of integrated circuits. Although there exist techniques such as the statistical process control (SPC) [2]-[3], statistical analysis methods [4]-[5] and machine learning methods [6] for monitoring the operations of the wafer probes, the probing errors may still occur in many aspects and cause some good dies being over killed; consequently, the profit is diminished. Fig. 1 shows the Causeand-Effect diagram of overkills.

Thus, minimizing the number of overkills is always one of the main goals in wafer probe testing process. The key tool to identify or save overkills is re-probe, which is an additional wafer probe testing. However, re-probe is a major factor for decreasing the throughput. Thus, the overkill and the re-probe possess inherent conflicting factors, because decreasing the former can gain more profit, however, at the expense of increasing the latter, which will degrade the throughput and increase the cost. What implies is that drawing a fine line for deciding whether to go for a re-probe to save possible overkills is an important research issue in this optimization problem of the wafer probe testing process. Considering the economic situation regarding throughput requirement, it would be most beneficial for us to use the trade-off method [7] to solve the current problem. That is to minimize the overkills subject to a tolerable level of re-probes provided by the decision maker.

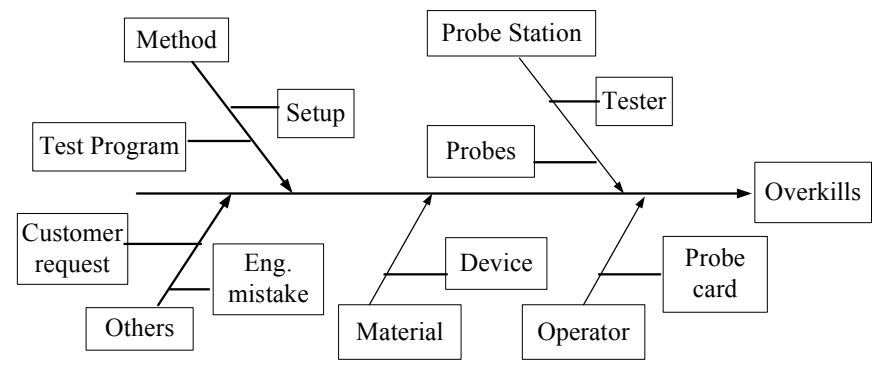

Figure 1. Cause-and-Effect diagram of overkills.

There may be various testing procedures in different chip manufacturers. After the wafer probe testing, a bin number is used to label each defective die of the wafer. A bin number denotes a classification of circuitry-defect failure in a die. The bin number goes from 1 to a certain number as defined by engineers. But, no matter what testing procedures are used, the decision for carrying out the re-probe should be based on whether the number of good dies and the number of defective dies in a wafer exceed the corresponding threshold values. Thus, determining these threshold values so as to minimize the overkills under a tolerable level of re-probes is the main theme of the optimization problem considered here. Furthermore, 
since the goodness of a die and the probing errors are of stochastic nature, the considered problem becomes a constrained stochastic simulation optimization problem.

Typical solution methods for such a type of problems may be the simulated annealing (SA) method [8], tabu search (TS) method [9], genetic algorithm (GA) [10], and evolutionary strategy (ES) [11]. The SA and TS methods have difficulties in handling the constraints. GA is a more popular technique than ES. However, calculating the fitness of a chromosome in the considered problem is very time consuming and cause GA to be very sluggish. This may prevent GA to obtain a good enough best-so-far solution within tolerable computation time. Thus, this computationally intractable problem is most suitable for the application of ordinal optimization (OO) theory [12][13] to seek for good enough threshold values.

We organize our paper in the following manner. In Section II, we will describe the constrained stochastic optimization problem of minimizing overkills under a tolerable level of reprobes in semiconductor wafer probe testing process. In Section III, we will present the ordinal optimization based algorithm and apply the proposed algorithm to the corresponding problem. In Section IV, we will show the test results of applying the proposed algorithm on a real case. We also demonstrate the solution quality and the computational efficiency by comparing with a vast number of randomly generated solutions and two competing methods, respectively. Finally, we will make a conclusion in Section V.

\section{PROBLEM STATEMENTS AND MATHEMATICAL FORMULATION}

There may be various testing procedures in different chip manufacturers. But no matter what testing procedures are used, the logic of the decision process for carrying out a retest are approximately the same. In this section, we employ typical testing procedures used in a local world-renowned wafer foundry in Taiwan. Fig. 2 shows the flow chart of the wafer probe testing procedures, the operation of which is briefly described in the following.

For every wafer, a wafer probe testing is performed twice. The second probe testing applies only to those dies failed in the first one. A die is considered to be good if it is good in either probe testing. We let $w_{i}\left(\bar{w}_{i}\right)$ denote the number of good (defective) dies in wafer $i$, and let $B_{i j}$ denote the number of bin $j$ in wafer $i$. Assume there are $J$ types of bins in a wafer, then $\bar{w}_{i}=\sum_{j=1}^{J} B_{i j}$ and $w_{i}=T D_{i}-\bar{w}_{i}$, where $T D_{i}$ denotes the total number of dies in wafer $i$. Following the two times of wafer probe testing, a two-stage checking on the number of good dies is performed to determine the necessity of carrying out a re-probe, i.e. an additional wafer probe testing. We let $W_{\min }$ denote the threshold value of the number of good dies in a wafer to determine whether to pass or hold the wafer; we let $b_{j \max }, j=1, \ldots, J$, denote the threshold value of the number of defective dies of bin $j$ in the hold wafer to determine whether to perform a re-probe. The mechanism of the two-stage checking can be summarized below. If $w_{i} \geq W_{\min }$, we pass wafer $i$; otherwise, we will hold this wafer and check its bins. Although we may pass the wafer when the threshold value test is a success, there may contain loss overkills. We let $V_{i}$ and $R_{i}$ denote the total number of loss overkills and re-probes in wafer $i$, respectively. Thus for the passed wafer $i$, we can calculate $V_{i}$ of wafer $i$. For the hold wafer $i$, if $B_{i j} \leq b_{j \max }$ for all $j$, then wafer $i$ will be passed and we can calculate $V_{i}$ of wafer $i$. For those hold wafers, if $B_{i j}>b_{j \max }$, we will perform re-probes for all defective dies of bin $j$ to check whether there are probing errors that cause overkills. Then, the overkills will be saved when there are probing errors and we can calculate $R_{i}$ of wafer $i$.

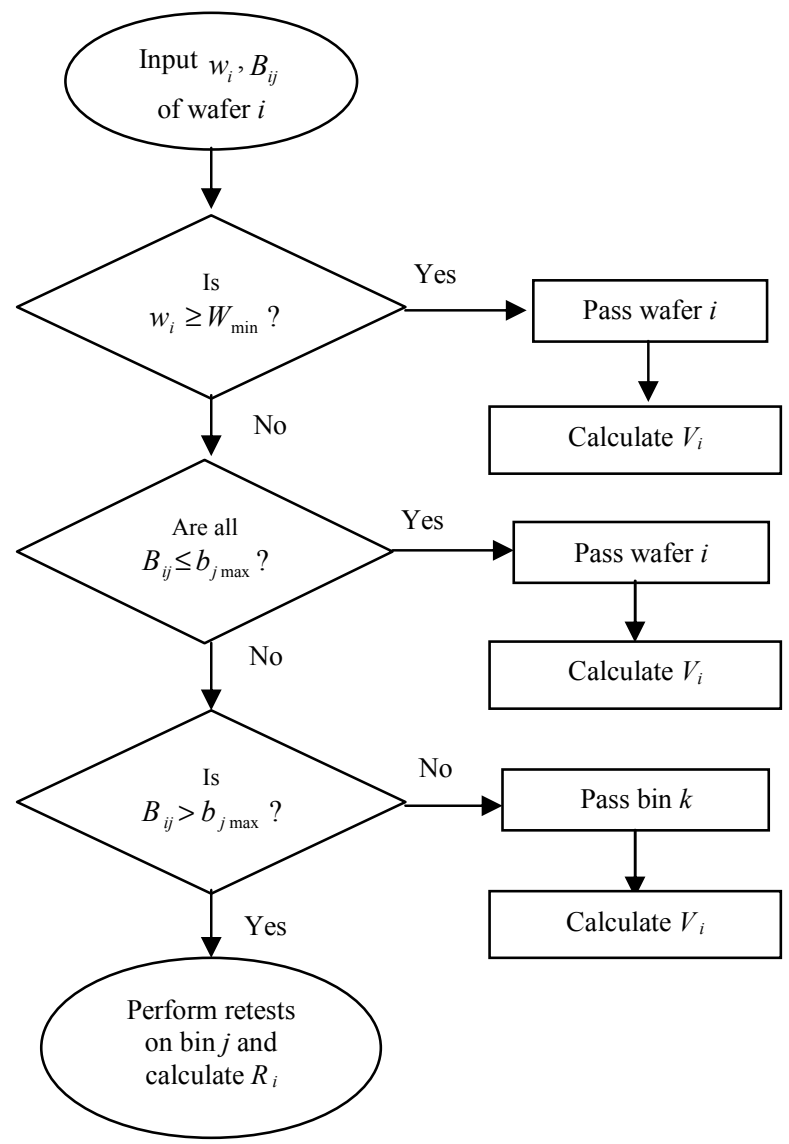

Figure 2. Flow chart of the wafer probe testing procedures.

This particular class of policies for deciding re-probe based on the threshold values is commonly practiced in wafer fabrication processes. Once all the threshold value tests for all bins of the hold wafer $i$ are completed, we can compute $V_{i}$ and $R_{i}$ of wafer $i$. The resulting values of $V_{i}$ and $R_{i}$ of wafer $i$ will be used to calculate $\bar{V}=\frac{1}{L} \sum_{i=1}^{L} V_{i}$ and $\bar{R}=\frac{1}{L} \sum_{i=1}^{L} R_{i}$, which represent the average overkills and re-probes per wafer, respectively, where $L$ denotes the total number of tested 
wafers. Thus, the relationship between the inputs and the outputs of the considered problem can be illustrated in Fig. 3, in which $W_{\min }, b_{j \max }, j=1, \ldots, J$, are the input variables, $\bar{V}$ and $\bar{R}$ are the output variables, and the total number of tested wafers $L$ is part of the testing procedures.

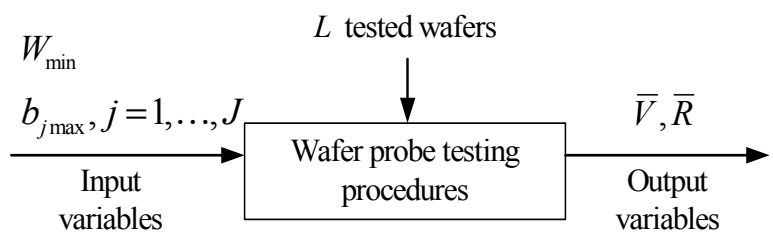

Figure 3. Relationship between the inputs and the outputs of wafer probe testing procedures.

We found that if we increase $W_{\min }$ while decreasing $b_{j \max }$, there will be more re-probes and less overkills. Thus, to minimize overkills under a tolerable level of re-probes, we will set minimizing the average number of overkills per wafer, $\bar{V}$, as our objective function while keeping the average number of re-probes per wafer, $\bar{R}$, under a satisfactory level. Thus, using the trade-off method, this optimization problem can be formulated as the following constrained stochastic simulation optimization problem:

$$
\begin{gathered}
\min _{x \in X} \bar{V} \\
\text { subject to } \bar{R} \leq r_{T}
\end{gathered}
$$

where $x=\left[W_{\min }, b_{j \max }, j=1, \ldots, J\right]$ denotes the vector of threshold values, that is the vector of input variables; $X$ denotes the input-variable space; and $r_{T}$ denotes the tolerable average-number of re-probes per wafer.

This constrained stochastic simulation optimization problem (1) is to find an optimal vector of threshold values, $x^{*}$, to minimize $\bar{V}$ subject to the employed testing procedures and the constraint on $\bar{R}$. Therefore, we can use a penalty function to transform (1) into the following unconstrained stochastic simulation optimization problem:

$$
\min _{x \in X} F(x)=\bar{V}+P\left(\bar{R}-r_{T}\right) \times\left(\bar{R}-r_{T}\right)
$$

where $P\left(\bar{R}-r_{T}\right)$ denotes a continuous penalty function for the constraint $\bar{R} \leq r_{T}$, such that $P\left(\bar{R}-r_{T}\right)>0$ for $\bar{R}>r_{T}$ and $P\left(\bar{R}-r_{T}\right)=0$ for $\bar{R} \leq r_{T}$. We used the sigmoid-type function as our penalty function $P\left(\bar{R}-r_{T}\right)=\eta \frac{1}{1+e^{-\left(\bar{R}-r_{T}\right)}}$ for $\bar{R}>r_{T}$, where $\eta$ is a normalized coefficient such that $\eta=\frac{\max _{i \in\{1, \ldots, L\}} \bar{V}_{i}}{\max _{i \in\{1, \ldots, L\}} \bar{R}_{i}}$, and $P\left(\bar{R}-r_{T}\right)=0$ for $\bar{R} \leq r_{T}$.

\section{ORDINAL OPTIMIZATION BASED ALGORITHM}

Since the order of vectors are relatively immune to effects of estimation noise, performance "order" of the vectors is likely to be preserved even evaluated using a crude model. Thus, to select $N$ good enough vectors from $X$ without consuming much computation time, we need to construct a crude but effective model to evaluate the objective value $F(x)$ for a given vector $x$, and use a selection scheme to select $N$ good enough vectors. The proposed crude model is constructed based on a shorter stochastic simulation with a small amount of test wafers, and the selection scheme is genetic algorithm (GA) [10].

\section{A. Genetic Algorithm}

To avoid an accurate but lengthy stochastic simulation to estimate $F(x)$ for a given vector $x$, a crude model is used to evaluate $F(x)$. The crude model is constructed basing on a shorter stochastic simulation with a small amount of test wafers $L_{0}$, say 1000 test wafers. As indicated in Chen et al. [14], the stochastic simulation of shorter length is an approximate model for the lengthy stochastic simulation. For the sake of simplicity in expression, we let $F_{0}(x)$ denote the objective value for a given $x$ computed by crude model with $L_{0}$ test wafers.

GA is a stochastic search algorithm based on the mechanism of natural selection and natural genetics. By the aid of the above effective objective value (or the so-called fitness value in GA terminology) evaluation model, we can select $N$ good enough vectors from $X$ using GA, which is briefly described as follows. Assuming an initial random population produced and evaluated, genetic evolution takes place by means of three basic genetic operators: (a) parent selection; (b) crossover; (c) mutation. The chromosome in GA terminology represents a vector $x$ in our problem, and each chromosome is encoded by a string of $0 \mathrm{~s}$ and $1 \mathrm{~s}$. Parent selection is a simple procedure whereby two chromosomes are selected from the parent chromosome based on their fitness values. Solutions with high fitness values have a high probability of contributing new offspring to the next generation. The selection rule we used in our approach is a simple roulette-wheel selection. Crossover is an extremely important operator for the GA. It is responsible for the structure recombination (information exchange between mating chromosomes) and the convergence speed of the GA and is usually applied with relatively high probability, say 0.8 . The chromosomes of the two parents selected are combined to form new chromosomes that inherit segments of information stored in parent chromosomes. There are many crossover scheme, we employ the single-point crossover in our approach. While crossover is the main genetic operator exploring the information included in the current generation, it does not produce new information. Mutation is the operator responsible for the injection of new information. With a small probability, random bits of the offspring chromosomes flip 
from 0 to 1 and vice versa and give new characteristics that do not exist in the parent chromosome. In our approach, the mutation operator is applied with a relatively small probability 0.03 to every bit of the chromosome.

There are two criteria for the convergence of GA. One is when the fitness value of the best chromosome does not improve from the previous generation, and the other is when evolving enough generations. In our algorithm, GA is iterated until the maximum iteration count $C_{\max }$ is reached. The initial populations of the GA are $I$, say 4000, randomly selected vectors from $X$. After the stopping criteria have been reached, we rank the final generation of these $I$ chromosomes based on their fitness values and pick the top $N$ chromosomes, which form the $N$ good enough vectors that we look for.

\section{B. Goal Softening Searching Procedures}

Starting from the selected $N$ good enough vectors, we will proceed directly with the existing goal softening searching procedures. That is, we will evaluate the objective value of each vector using a more refined model than the crude one employed in GA. This more refined model uses stochastic simulation with various number of test wafers $L$. We let $L_{s}=10^{6}$ represent the sufficiently large $L$. In the sequel, we define the exact model of (2) as when the test wafers $L=L_{s}$. For the sake of simplicity in expression, we let $F_{s}(x)$ denote the objective value of a vector $x$ computed by exact model, i.e. $L=L_{s}$.

First, we define an appropriate basic number of test wafers $L_{0}=1000$. We set the number of test wafers in subphase $i$, denoted by $L_{i}$, to be $L_{i}=e L_{i-1}$ (or $\left.L_{i}=e^{i} L_{0}\right), i=1,2, \ldots$ We let $N_{1}=N$ and set the size of the selected estimated good enough subset in subphase $i$ to be $N_{i}=N_{i-1} / e$ (or $N_{i}=N_{1} / e^{i-1}$ ), $i=2,3, \ldots$. If the obtained value of $N_{i}$ is not an integer, we round it to the nearest integer. We denote $n_{s}$ as the total number of subphases, and $n_{s}$ is determined by

$$
n_{s}=\arg \left\{\min _{n_{s}}\left(L_{0} e^{n_{k}-1} \leq L_{s}<L_{0} e^{n_{k}}, 1 \leq N_{n_{s}}<10\right)\right\}
$$

where $L_{s}=10^{6}$. The above formula determines $n_{s}$ to be the minimum of the following: (i) the $n_{s}$ such that test wafers $L_{0} e^{n_{s}}$ exceeds the length of exact model, $L_{s}$, and (ii) the size of the selected estimated good enough subset resulted in subphase $n_{s}$ is small enough, i.e. $N_{n_{s}}<10$. Once $n_{s}$ is determined, from subphase $i=1$ to $n_{s}-1$, we use the stochastic simulation with the number of test wafers $L_{i}=e^{i} L_{0}$ to estimate the $F(x)$ of the $N_{i}=N / e^{i-1}$ candidates in subphase $i$, then we rank the $N / e^{i-1}$ candidates based on their estimated $F(x)$ and select the best $N / e^{i}$ candidates as the candidate solution set for subphase $i+1$. Finally, we use a stochastic simulation with the number of test wafers $L_{s}$ to compute $F_{s}(x)$ of the $N_{n_{s}}$ solutions in the last subphase (i.e. subphase $n_{s}$ ). In fact, the more refined model is in fact the accurate model of (2) in the last subphase, and the vector with smallest $F_{s}(x)$ is the good enough vector that we seek.

\section{The OO based algorithm}

Now, our OO based algorithm can be stated as follows.

Step 1: Randomly select $I$ vectors from $X$ as the initial populations. Apply a GA with the following setup: simple roulette-wheel selection scheme, single-point crossover scheme with probability $p_{c}$, and mutation probability $p_{m}$ to these chromosomes by the aid of the fitness-value evaluated rough model using a small amount of test wafers. After the algorithm reaches the maximum iteration $C_{\max }$, we rank all the final $I$ chromosomes based on their fitness values and select the best $N$ chromosomes (i.e. $x$ 's).

Step 2: Define an appropriate basic number of test wafers $L_{0}$ and determine the number of subphases $n_{s}$ by (3).

Step 3: Use the stochastic simulation with test wafers $L_{i}=e^{i} L_{0}$ to estimate $F(x)$ of the candidate $N / e^{i-1}$ $x$ 's, $i=1, \ldots, n_{s}-1$; rank the candidate $N / e^{i-1} x$ 's based on their estimated $F(x)$ and select the best $N / e^{i} x$ 's as the candidate solution set for subphase $i+1$.

Step 4: Use the stochastic simulation with test wafers $L_{s}$ to compute the $F_{s}(x)$ of the candidate $N / e^{n_{s}-1} x$ 's. The $x$ with the smallest $F_{s}(x)$ is the good enough $x$ that we look for.

\section{TEST RESULtS}

Our simulations are based on the following data collected from a practical product containing 630 test wafers of a renowned wafer foundry in Taiwan. The product is made in 6inches wafers. Each wafer consists of 206 dies. There are 10 bins in the wafers of this product, and the values of their means $\mu_{j}, j=1, \ldots, 10$, are respectively the following 10 positive real numbers: $0.5,0.5,1.1,1.3,0.8,3.7,3.5,40,45$, and 13 . The yield rate of this product is $46.6 \%$. The mean of the overkills that occurred in bin $j$ is $0.03 \times \mu_{j}$ for $j=1, \ldots, 10$, that is $\tau_{j}=0.03$ for all $j$. The normalized coefficient $\eta$ in the continuous penalty function is 0.0617 . The input-variable space is $X=\left\{x=\left[W_{\min }, b_{j \max }, j=1, \ldots, 10\right] \mid\right.$ $\left.W_{\min } \in[1,206], b_{j \max } \in[1,206], j=1, \ldots, 10\right\}$. We have simulated three cases of different $r_{T}$ 's, which are 20, 50 and 80 . 
Specific data in the OO based algorithm applying to this product are given in the following. First, we narrow down the decision-variable space $X$ by excluding the irrational threshold values and denote the reduced decision variable space by ^ $\hat{X}=\left\{x=\left[W_{\min }, b_{j \max }, j=1, \ldots, 10\right] \mid W_{\min } \in[50,206]\right.$,

$\left.b_{j \max } \in\left[1,4 \mu_{j}\right], j=1, \ldots, 10\right\}$. In Step 1, the parameters are set as $I=4000, \quad N=1000, \quad p_{c}=0.8, \quad p_{m}=0.03$, and the convergence criteria we employed for our GA is when the evolving number of generations exceed $C_{\max }=30$. In Step 2, we define an appropriate basic number of test wafers $L_{0}=1000$ and the number of subphases $n_{s}$ is 6 . Table 1 shows the number of candidate solutions and test wafers in each subphase of Step 3.

TABle 1. Number of CANDidate Solutions and Test Wafers in EACH SUBPHASE.

\begin{tabular}{|c|c|c|c|c|c|c|}
\hline Subphase $i$ & 1 & 2 & 3 & 4 & 5 & 6 \\
\hline$N_{i}$ & 1000 & 368 & 135 & 50 & 18 & 7 \\
\hline$L_{i}$ & 2718 & 7388 & 20079 & 54575 & 148334 & $10^{6}$ \\
\hline
\end{tabular}

The good enough vector of threshold values and the average overkill percentage for three cases $r_{T}=20,50$ and 80 we obtained from the $\mathrm{OO}$ based algorithm are shown in Table 2. From this table, we can observe that when $r_{T}$ increases, the values of $W_{\min }$ increases as shown in row 2 , and the values of leading $b_{j \max }, j=8$ and 9, which accounts for most of the reprobes, decrease as shown in rows 10 and 11 , respectively. This indicates that if we allow more re-probes, that is increasing $r_{T}$, we can set more stringent threshold values, that are increasing $W_{\min }$ and decreasing the leading $b_{j \max }$, so as to save more overkills, that is decreasing the average overkill percentage, as indicated in the last row of Table 2. This also demonstrates the conflicting nature between the two objectives - minimizing overkills under a tolerable level of re-probes.

We also use the 630 real test wafers, whose bins and overkills before re-probe are known, to test the performance of the vector of threshold values obtained by our algorithm for the three cases shown in Table 2. The corresponding results of the pair of the average overkills per wafer, $\bar{V}\left(=\frac{1}{630} \sum_{i=1}^{630} V_{i}\right)$, and the average re-probes per wafer, $\bar{R}\left(=\frac{1}{630} \sum_{i=1}^{630} R_{i}\right)$, for these 630 test wafers are shown in Fig. 4 as the points marked by “之े", "०”, "*” with the corresponding $r_{T}$ shown on the top right corner of the figure. We also use 3000 randomly selected vectors of threshold values to test the same 630 test wafers; the resulted pairs of $\bar{V}$ and $\bar{R}$ are shown as the points marked by "॰" in Fig. 4.

We have also used typical GA and ES methods to solve the exact model of problem (2) using $L_{s}$ test wafers for the case of $r_{T}=50$. As indicated in Section 1, the global searching techniques are computationally expensive in solving the exact model of (2). In Fig. 5, we show the progress of the best-so-far objective values of (2) versus the CPU times consumed by the GA and ES methods. From this figure, we can observe the sluggish improvement of the best-so-far objective values of these two methods. We stop the GA and ES methods when they consumed 60 times of the CPU time consumed by the $\mathrm{OO}$ based algorithm, and the best-so-far objective values of (2) they obtained are still $16.61 \%$ and $25.46 \%$ more than the final objective value obtained by the $\mathrm{OO}$ based algorithm, respectively. Using the threshold values they obtained to test the 630 wafers, the resulted $(\bar{V}, \bar{R})$ pairs from GA and ES methods are marked by "+" and " $\Delta$ " in Fig. 4 , respectively. We found that using OO based algorithm, we can save $17.56 \%$ and $26.77 \%$ more overkills than using the GA and ES methods for $\bar{R} \leq 50$, respectively. In addition, both GA and ES methods do not generate the optimal solution, because the best so far solution they obtained for one hour of CPU time are still far away from the optimal solution of (2).

We see that for $\bar{R} \leq 50$, the $\bar{V}$ resulted by the good enough vectors of threshold values obtained by our algorithm is almost the minimum compared with the randomly selected vectors of threshold values. Similar conclusions can be drawn for the cases of $r_{T}=20$ and 80 . From Fig. 4, we can see that the results we obtained for the cases of $r_{T}=20,50$ and 80 are almost on the boundary of the region resulted from the randomly generated vectors of threshold values; this implicit boundary represents the $(\bar{V}, \bar{R})$ pairs resulted by the optimal vectors of threshold values. The above result implies that our algorithm not only controls the level of re-probes but also obtain a near optimal solution.

TABle 2. The Good Enough Vector of Threshold Values and The Average Overkill Percentage for Three DifFerent $r_{T}{ }^{\text {'S }}$.

\begin{tabular}{|c|c|c|c|}
\hline $\begin{array}{l}\text { Good } \\
\text { enough vector }\end{array}$ & 20 & 50 & 80 \\
\hline$W_{\min }$ & 109 & 123 & 141 \\
\hline$b_{1 \max }$ & 2 & 1 & 1 \\
\hline$b_{2 \max }$ & 2 & 2 & 1 \\
\hline$b_{3 \max }$ & 3 & 3 & 3 \\
\hline$b_{4 \max }$ & 3 & 4 & 2 \\
\hline$b_{5 \max }$ & 2 & 1 & 1 \\
\hline$b_{6 \max }$ & 8 & 7 & 7 \\
\hline$b_{7 \max }$ & 7 & 5 & 6 \\
\hline$b_{8 \max }$ & 59 & 43 & 31 \\
\hline$b_{9 \max }$ & 71 & 58 & 49 \\
\hline$b_{10 \max }$ & 28 & 16 & 18 \\
\hline$\frac{\bar{V}}{206} \times 100 \%$ & $1.18 \%$ & $0.68 \%$ & $0.22 \%$ \\
\hline \hline
\end{tabular}




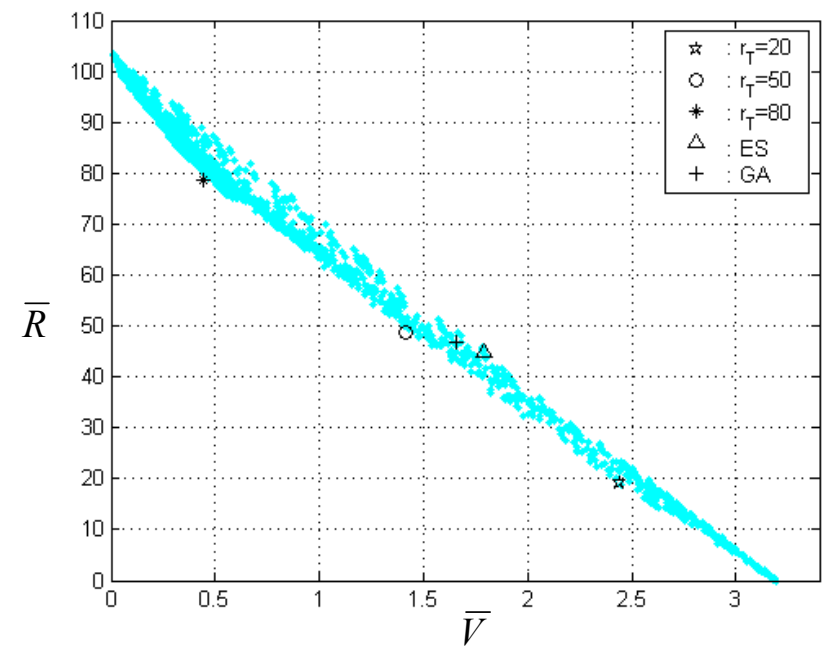

Figure 4. The resulted pairs of $(\bar{V}, \bar{R})$ obtained by our algorithm and the randomly generated vector of threshold values.

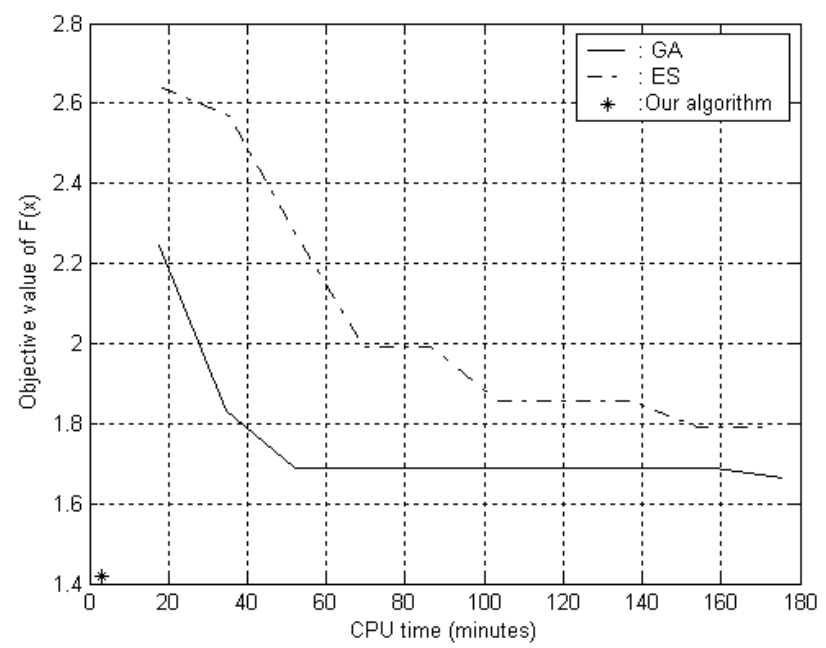

Figure 5. Comparison of the computational efficiency and the quality of the obtained solution by our algorithm, the GA and ES methods.

\section{CONCLUSION}

To cope with the computationally intractable constrained stochastic simulation optimization problems, we have proposed an ordinal optimization based algorithm to solve for a good enough solution using reasonable computational time. To demonstrate the applicability of the proposed algorithm, we have used it to solve for a vector of good enough threshold values to minimize overkills under a tolerable level of reprobes in a wafer probe testing process of a wafer foundry. We have tested the performance of the solution we obtained using the real data and found that the resulting average number of overkills and re-probes per wafer lie almost on the boundary resulted from the optimal vector of threshold values of the considered problem. We have demonstrated the computational efficiency of the proposed algorithm by comparing with the genetic algorithm and the evolution strategy. The vector of good enough threshold values obtained by the proposed algorithm is promising in the aspects of solution quality and computational efficiency.

\section{ACKNOWLEDGMENT}

This research work is supported in part by the National Science Council in Taiwan, R.O.C., under grant NSC98-2221E-324-039.

\section{REFERENCES}

[1] T.S. Barnett, M. Grady, K. Purdy, and A.D. Singh, "Exploiting prediction defect clustering for yield and reliability," IEE Proceedings-Computers And Digital Techniques, vol. 152, no. 3, pp. 407-413, 2005.

[2] S. Muriel, P. Garcia, O. Marie-Richard, M. Monleon, and M. Recio, "Statistical bin analysis on wafer probe," in Proceedings of the 2001 IEEE/SEMI Advanced Semiconductor Manufacturing Conference and Workshop, Munich, Germany, 2001, pp. 187-192.

[3] K.R. Skinner, D.C. Montgomery, G.C. Runger, J.W. Fowler, D.R McCarville, T.R. Rhoads, and J.D. Stanley, "Multivariate statistical methods for modeling and analysis of wafer probe test data," IEEE Transactions on Semiconductor Manufacturing, vol. 15, no. 4, pp. 523$530,2002$.

[4] S. Sabade, and D.M.H. Walker, "Improved wafer-level spatial analysis for IDDQ limit setting," In: Proceedings of the IEEE International Test Conference 2001, Baltimore, MD, 2001, pp. 82-91.

[5] F.L. Chen, S.C. Lin, Y.Y. Doong, and K.L. Young, "LOGIC product yield analysis by wafer bin map pattern recognition supervised neural network," in Proceedings of the 2003 IEEE International Symposium on Semiconductor Manufacturing, San Jose, CA, 2003, pp. 501-504.

[6] S.C. Hsu, and C.F. Chien, "Hybrid data mining approach for pattern extraction from wafer bin map to improve yield in semiconductor manufacturing," International Journal of Production Economics, vol. 107, no. 1, pp. 88-103, 2007.

[7] Y. Collette, and P. Siarry, Multiobjective optimization: principles and case studies. New York: Springer-Verlag, 2003.

[8] B. Suman, and P. Kumar, "A survey of simulated annealing as a tool for single and multiobjective optimization," Journal of the Operational Research Society, vol. 57, no. 10, pp. 1143-1160, 2006.

[9] A.R. Hedar, and M. Fukushima, "Tabu Search directed by direct search methods for nonlinear global optimization," European Journal of Operational Research, vol. 17, no. 3, pp. 329-349, 2006.

[10] S.N. Sivanandam, and S.N. Deepa, Introduction to genetic algorithms. Springer, Berlin Heidelberg, 2008.

[11] H.G. Beyer, The theory of evolution strategies. Natural Computing Series. Springer, Heidelberg, 2001.

[12] Y.C. Ho, "An explanation of ordinal optimization: Soft computing for hard problems," Information Sciences, vol. 113, no. 3-4, pp. 169-192, 1999.

[13] Y.C. Ho, Q.C. Zhao, and Q.S. Jia, Ordinal optimization: Soft optimization for hard problems. New York: Springer-Verlag, 2007.

[14] C.H. Chen, S.D. Wu, and L. Dai, "Ordinal comparison of heuristic algorithms using stochastic optimization," IEEE Transactions on Robotics and Automation, vol. 15, no. 1, pp. 44-56, 1999. 